\title{
INFLUENCE OF ORGANIZATIONAL CULTURE AND FINANCIAL STRATEGY ON EMPLOYEE PERFORMANCE IN PT. SILOAM INTERNATIONAL HOSPITALS MANADO
}

\author{
Raymond Rombot, Jufry Rompas, Jeane Christine Lasut, Denny I.Y Rompas \\ Department Of Accounting, Polytechnic of Manado Country \\ DOI: $10.31364 / \mathrm{SCIRJ} / \mathrm{v6.i9} 9.2018 . P 0918560$ \\ http://dx.doi.org/10.31364/SCIRJ/v6.i9.2018.P0918560
}

\begin{abstract}
The purpose of this research to determine the effect between dependent and independent variables. The research was carried out on finance accounting employees at PT Siloam International Hospitals Manado, with a total of 30 respondents. Data collection was carried out using questionnaires, interviews, and direct observation in the field. Data collected and analyzed by statistical analysis using Multiple Linear Regression Test. The results showed that there was a positive and significant influence on the financial strategy $0.003<0.05$ on the performance of employees in finance accounting and there was a joint influence of $0.000<0.05$ between the variables of organizational culture and financial strategies on the performance of employees of finance accounting at PT Siloam International Hospitals Manado. The most dominant factor influencing employee performance is financial strategy, this is evidenced by the highest standardized coefficient value. Financial strategies have a positive and significant effect on employee performance. The better the financial strategy the employee's performance will increase. While organizational culture does not have a positive effect on employee performance. This means that if the organizational culture gets better then employee performance will increase. It is expected that with improvements in company policy and financial strategies can maintain existing indicators and future so that employees can improve their performance.
\end{abstract}

Keywords: Organizational Culture, Financial Strategy, Employee Performance.

\section{Introduction}

A good organization is a key factor to improve employee performance. Improving employee performance will bring progress for the company to survive in an unstable business competition. Therefore, efforts to improve employee performance are the most serious management challenges because the success of the company's survival depends on the quality of the human resource performance in it. Coaching and developing new employees, or long time in the company is one of the activities in order to adjust to changes and development of employees.

Competitive employee development companies need high-achieving employees. At the same time workers need feedback on their performance as a guide for their actions in the future. High employee performance is expected by the company. The more employees who have high performance, the overall productivity of the company will increase, so that the company will survive in global competition. Employees are required to be able to complete their tasks and responsibilities effectively and efficiently. Employee success can be measured through customer satisfaction, reduced number of complaints, and achievement of optimal targets. Efforts to improve employee performance, namely organizational culture, and financial strategies, or planning to increase company profits. The formation process, in the end will help in producing capable, high integrity individuals. If the organizational culture, and the company's financial strategy are bad, then things that are not desirable will occur, namely accounting fraud.

Accounting fraud can be prevented if there is a high commitment not to commit various forms of fraud, management or other parties involved. Moral crisis at the moment, is indeed a major problem and various ways can indeed be taken to detect and prevent fraud, including by streamlining internal control, law enforcement, implementing good governance. In an effort to realize optimal and quality health services, the role of the hospital becomes very important. Hospital management in managing its business is required to operate professionally. Hospital management professionalism needs to be supported by all parties by following the development of technology and knowledge and the use of resources can be pursued effectively and efficiently. Organizational culture in the hospital seen from the level of commitment of hospital members, or employees towards values and beliefs from leaders to all levels of employees. The factors of values and basic beliefs play a role in shaping the ethics, attitudes, behavior of members of the 
organization, and shape their perspective on problems both internal and external faced in organizational life. However, there are often employees who are not disciplined do not obey the rules in the organization so the organizational culture is depicted as unstable and bad it is very detrimental to the hospital. The financial strategy in the hospital must be planned in the form of financial management, which is in accordance with the rules in the hospital. Hospital management, not only lies in one financial function, as we have understood so far. However, related to all functions of the hospital, both service functions and service support. This makes it important to understand several aspects of financial management in hospital management.

This research focuses on Finance and Accounting employees at Siloam International Hospitals located in Manado. Success or failure of employees in work performance can be influenced by organizational culture. Organizational culture can be functioned as a demand that binds its members because it can be formulated formally in various rules and regulations of the organization so that individuals within the organization will indirectly be bound so as to form attitudes and behaviors that are in accordance with the vision and mission of the organization . Based on the description above, the researcher tries to analyze how much influence the organizational culture, financial strategies on employee performance finance and accounting.

\section{RESEARCH METHODS}

The research used by the author is descriptive research. Descriptive analysis method is research conducted to determine the value of variables either one variable, or more without making comparisons, or connecting with other variables. Data collection in this study is interviews and questionnaires for primary data collection and document review for secondary data. There were 30 respondents both interviews and questionnaires. The interviews were conducted by the researchers themselves, while the questionnaires were filled directly by employees of PT Siloam International Hospitals Manado. This research is also a quantitative research. That is the measurement of quantitative and objective statistical data through scientific calculations derived from samples of people or residents who were asked to answer a number of questions about the survey or to determine the frequency and the percentage of respondents' responses.

\section{DISCUSSION}

\subsection{Variable Operational Description}

\section{Independent Variables}

Independent variables are types of variables that explain or affect other variables. Independent variables in this study, namely:

a. Organizational culture $\left(\mathrm{x}_{1}\right)$

b. Financial Strategy $\left(\mathrm{x}_{2}\right)$

\section{Dependent Variables}

The dependent variable is the variable described, or is influenced by the independent variable. The dependent variable in this research employee performance $(\mathrm{Y})$

\subsection{Questionnaire results}

\section{Descriptive Analysis of Variables}

Descriptive statistical analysis is intended to determine the frequency distribution of respondents' answers to the results of the questionnaire distributed. The results of the questionnaire include organizational culture variables (X1), financial strategies (X2), and the dependent variable performance of financial accounting employees (Y).

\section{a. Organizational Culture $\left(\mathbf{X}_{1}\right)$}

Based on the data collected from the questionnaire on organizational culture, it can be seen that the number of scores from these variables is shown in the following table.. 
Table 3.1 Score X1 Respondents on the Questionnaire

\begin{tabular}{|c|c|c|c|c|c|c|}
\hline \multirow{3}{*}{$\begin{array}{c}\text { Num } \\
\text { ber }\end{array}$} & \multirow{3}{*}{ Information } & \multicolumn{5}{|c|}{ Score } \\
\hline & & $\begin{array}{l}\text { respondents } \\
\text { (SD) }\end{array}$ & $\begin{array}{l}\text { respondents } \\
\text { (D) }\end{array}$ & $\begin{array}{l}\text { respondents } \\
\text { (d) }\end{array}$ & $\begin{array}{l}\text { respondents } \\
\text { (A) }\end{array}$ & $\begin{array}{l}\text { respondents } \\
\text { (SA) }\end{array}$ \\
\hline & & 1 & 2 & 3 & 4 & 5 \\
\hline 1 & $\begin{array}{c}\text { X1. Statement } 1 \\
\text { I use work uniforms according to the } \\
\text { provisions }\end{array}$ & $\begin{array}{c}1 \\
(3,33)\end{array}$ & $\begin{array}{c}2 \\
(6,67)\end{array}$ & $\begin{array}{c}2 \\
(6,67)\end{array}$ & $\begin{array}{c}14 \\
(46,67 \%)\end{array}$ & $\begin{array}{c}11 \\
(36,67 \%)\end{array}$ \\
\hline 2 & $\begin{array}{c}\text { X1. Statement } 2 \\
\text { I always ask permission from my } \\
\text { supervisor if there are activities } \\
\text { outside the office }\end{array}$ & $\begin{array}{c}0 \\
(0)\end{array}$ & $\begin{array}{c}5 \\
(16,67 \%)\end{array}$ & $\begin{array}{c}4 \\
(13,33)\end{array}$ & $\begin{array}{c}16 \\
(53,33)\end{array}$ & $\begin{array}{c}5 \\
(16,67 \%)\end{array}$ \\
\hline 3 & $\begin{array}{c}\text { X1. Statement } 3 \\
\text { I choose a job that needs to be } \\
\text { prioritized, so that everything can be } \\
\text { completed. }\end{array}$ & $\begin{array}{c}1 \\
(3,33)\end{array}$ & $\begin{array}{c}1 \\
(3,33)\end{array}$ & $\begin{array}{c}3 \\
(10 \%)\end{array}$ & $\begin{array}{c}15 \\
(50 \%)\end{array}$ & $\begin{array}{c}10 \\
(33,33 \%)\end{array}$ \\
\hline 4 & $\begin{array}{c}\text { X1. Statement } 4 \\
\text { Assessment of work performance can } \\
\text { strengthen the working relationship } \\
\text { between leaders and subordinates. }\end{array}$ & $\begin{array}{c}1 \\
(3,33)\end{array}$ & $\begin{array}{c}1 \\
(3,33)\end{array}$ & $\begin{array}{c}1 \\
(3,33)\end{array}$ & $\begin{array}{c}12 \\
(40 \%)\end{array}$ & $\begin{array}{c}15 \\
(50 \%)\end{array}$ \\
\hline 5 & $\begin{array}{c}\text { X1. Statement } 5 \\
\text { Management always pays attention to } \\
\text { employees. }\end{array}$ & $\begin{array}{c}2 \\
(6,67)\end{array}$ & $\begin{array}{c}1 \\
(3,33)\end{array}$ & $\begin{array}{c}6 \\
(20 \%)\end{array}$ & $\begin{array}{c}17 \\
(56,67 \%)\end{array}$ & $\begin{array}{c}3 \\
(10 \%)\end{array}$ \\
\hline 6 & $\begin{array}{c}\text { X1. Statement } 6 \\
\text { Employees are given sufficient } \\
\text { facilities. }\end{array}$ & $\begin{array}{c}1 \\
(3,33)\end{array}$ & $\begin{array}{c}2 \\
(6,67)\end{array}$ & $\begin{array}{c}10 \\
(33,33 \%)\end{array}$ & $\begin{array}{c}16 \\
(53,33 \%)\end{array}$ & $\begin{array}{c}1 \\
(3,33)\end{array}$ \\
\hline 7 & $\begin{array}{c}\text { X1. Statement } 7 \\
\text { Give a salary according to the job }\end{array}$ & $\begin{array}{c}3 \\
(10 \%)\end{array}$ & $\begin{array}{c}5 \\
(16,67 \%)\end{array}$ & $\begin{array}{c}2 \\
(6,67 \%)\end{array}$ & $\begin{array}{c}15 \\
(50 \%)\end{array}$ & $\begin{array}{c}5 \\
(16,67 \%)\end{array}$ \\
\hline 8 & $\begin{array}{c}\text { X1. Statement } 8 \\
\text { Given the opportunity to express } \\
\text { opinions in meetings }\end{array}$ & $\begin{array}{c}3 \\
(10 \%)\end{array}$ & $\begin{array}{c}2 \\
(6,67)\end{array}$ & $\begin{array}{c}7 \\
(23,33 \%)\end{array}$ & $\begin{array}{c}12 \\
(40 \%)\end{array}$ & $\begin{array}{c}6 \\
(20 \%)\end{array}$ \\
\hline 9 & $\begin{array}{c}\text { X1. Statement } 9 \\
\text { New employees only need a few days } \\
\text { to adjust. }\end{array}$ & $\begin{array}{c}5 \\
(16,67)\end{array}$ & $\begin{array}{c}3 \\
(10 \%)\end{array}$ & $\begin{array}{c}4 \\
(13,33 \%)\end{array}$ & $\begin{array}{c}11 \\
(36,67 \%)\end{array}$ & $\begin{array}{c}8 \\
(26,67)\end{array}$ \\
\hline 10 & $\begin{array}{c}\text { X1. Statement } 10 \\
\text { If social activities, such as giving } \\
\text { donations to an orphanage, I become } \\
\text { generous. }\end{array}$ & $\begin{array}{c}1 \\
(3,33 \%)\end{array}$ & $\begin{array}{c}5 \\
(16,67)\end{array}$ & $\begin{array}{c}9 \\
(30 \%)\end{array}$ & $\begin{array}{c}11 \\
(36,67 \%)\end{array}$ & $\begin{array}{c}4 \\
(13,33 \%)\end{array}$ \\
\hline 11 & $\begin{array}{c}\text { X1. Statement } 11 \\
\text { In the office, colleagues argue that I } \\
\text { am diligent. }\end{array}$ & $\begin{array}{c}2 \\
(6,67 \%)\end{array}$ & $\begin{array}{c}6 \\
(20 \%)\end{array}$ & $\begin{array}{c}5 \\
(16,67 \%)\end{array}$ & $\begin{array}{c}15 \\
(50 \%)\end{array}$ & $\begin{array}{c}5 \\
(16,67)\end{array}$ \\
\hline 12 & $\begin{array}{c}\text { X1. Statement } 12 \\
\text { In making a decision, does the } \\
\text { company always consider the } \\
\text { aspirations of workers }\end{array}$ & $\begin{array}{c}4 \\
(13,33 \%)\end{array}$ & $\begin{array}{c}1 \\
(3,33)\end{array}$ & $\begin{array}{c}6 \\
(20 \%)\end{array}$ & $\begin{array}{c}15 \\
(50 \%)\end{array}$ & $\begin{array}{c}4 \\
(13,33 \%)\end{array}$ \\
\hline 13 & $\begin{array}{c}\text { X1. Statement } 13 \\
\text { Our organization produces something } \\
\text { that people need }\end{array}$ & $\begin{array}{c}3 \\
(10 \%)\end{array}$ & $\begin{array}{c}2 \\
(6,67 \%)\end{array}$ & $\begin{array}{c}2 \\
(6,67 \%)\end{array}$ & $\begin{array}{c}12 \\
(40 \%)\end{array}$ & $\begin{array}{c}9 \\
(30 \%)\end{array}$ \\
\hline 14 & $\begin{array}{l}\text { X1. Statement } 14 \\
\text { Our organization always encourages } \\
\text { us, to help overcome community } \\
\text { problems, and the environment in } \\
\text { which we work. }\end{array}$ & $\begin{array}{c}0 \\
(0)\end{array}$ & $\begin{array}{c}2 \\
(6,67 \%)\end{array}$ & $\begin{array}{c}4 \\
(13,33 \%)\end{array}$ & $\begin{array}{c}11 \\
(36,67 \%)\end{array}$ & $\begin{array}{c}13 \\
(43,33 \%)\end{array}$ \\
\hline 15 & $\begin{array}{c}\text { X1. Statement } 15 \\
\text { The organization always encourages } \\
\text { us, to help overcome the problems of }\end{array}$ & $\begin{array}{c}2 \\
(6,67 \%)\end{array}$ & $\begin{array}{c}3 \\
(10 \%)\end{array}$ & $\begin{array}{c}11 \\
(36,67)\end{array}$ & $\begin{array}{c}11 \\
(36,67)\end{array}$ & $\begin{array}{c}3 \\
(10 \%)\end{array}$ \\
\hline
\end{tabular}




\begin{tabular}{|c|c|c|c|c|c|c|}
\hline & $\begin{array}{l}\text { the community, and the environment } \\
\text { in which we work. }\end{array}$ & & & & & \\
\hline \multirow{3}{*}{ No } & \multirow{3}{*}{ Information } & \multicolumn{5}{|c|}{ Skor } \\
\hline & & $\begin{array}{l}\text { respondents } \\
\text { (SD) }\end{array}$ & $\begin{array}{l}\text { respondents } \\
\text { (D) }\end{array}$ & $\begin{array}{l}\text { respondents } \\
\text { (d) }\end{array}$ & $\begin{array}{l}\text { respondents } \\
\text { (A) }\end{array}$ & $\begin{array}{l}\text { respondents } \\
\text { (SA) }\end{array}$ \\
\hline & & 1 & 2 & 3 & 4 & 5 \\
\hline 16 & $\begin{array}{c}\text { X1. Statement } 16 \\
\text { Our organization dominates all } \\
\text { activities in the work environment }\end{array}$ & $\begin{array}{c}2 \\
(6,67 \%)\end{array}$ & $\begin{array}{c}1 \\
(3,33 \%)\end{array}$ & $\begin{array}{c}1 \\
(3,33 \%)\end{array}$ & $\begin{array}{c}20 \\
(66,67 \%)\end{array}$ & $\begin{array}{c}6 \\
(20 \%)\end{array}$ \\
\hline 17 & $\begin{array}{c}\text { X1. Statement 17 } \\
\text { Organizations always give } \\
\text { opportunities, to correct mistakes that } \\
\text { have been made, because in my } \\
\text { opinion it is a valuable lesson. }\end{array}$ & $\begin{array}{c}1 \\
(3,33 \%)\end{array}$ & $\begin{array}{c}2 \\
(6,67 \%)\end{array}$ & $\begin{array}{c}4 \\
(13,33 \%)\end{array}$ & $\begin{array}{c}15 \\
(50 \%)\end{array}$ & $\begin{array}{c}7 \\
(23,33 \%)\end{array}$ \\
\hline \multirow[t]{2}{*}{18} & $\begin{array}{c}\text { X1. Statement } 18 \\
\text { Assessment of one's performance } \\
\text { must be related to other aspects such } \\
\text { as honesty, cooperation, absenteeism } \\
\text { and others }\end{array}$ & $\begin{array}{c}2 \\
(6,67)\end{array}$ & $\begin{array}{c}2 \\
(6,67 \%)\end{array}$ & $\begin{array}{c}3 \\
(10 \%)\end{array}$ & $\begin{array}{c}9 \\
(30 \%)\end{array}$ & $\begin{array}{c}14 \\
(46,67 \%)\end{array}$ \\
\hline & $\begin{array}{l}\text { Average } \\
\end{array}$ & \multicolumn{4}{|c|}{$66,83 \%$} & Good \\
\hline
\end{tabular}

Source: processed data, 2018

Information

Strongly Disagree (SD)

Disagree (D)

Doubtful (d)

Agree (A)

Strongly Agree (SA)

\section{b. Financial Strategy $\left(\mathbf{X}_{2}\right)$}

Based on the data collected from the questionnaire about financial strategy, it appears that the number of scores from these variables is shown in the following table:

Table 3.2. Score $X_{2}$ Respondents on the Questionnaire

\begin{tabular}{|c|c|c|c|c|c|c|}
\hline \multirow{3}{*}{ No } & \multirow{3}{*}{ Information } & \multicolumn{5}{|c|}{ Skor } \\
\hline & & $\begin{array}{l}\text { respondents } \\
\text { (SD) }\end{array}$ & $\begin{array}{l}\text { respondents } \\
\text { (D) }\end{array}$ & $\begin{array}{l}\text { respondents } \\
\text { (d) }\end{array}$ & $\begin{array}{l}\text { respondents } \\
\text { (A) }\end{array}$ & $\begin{array}{l}\text { respondents } \\
\text { (SA) }\end{array}$ \\
\hline & & 1 & 2 & 3 & 4 & 5 \\
\hline 1 & $\begin{array}{c}\text { X2. Statement } 1 \\
\text { Streamline monitoring and control } \\
\text { systems. }\end{array}$ & $\begin{array}{c}0 \\
(0)\end{array}$ & $\begin{array}{c}0 \\
(0)\end{array}$ & $\begin{array}{c}0 \\
(0)\end{array}$ & $\begin{array}{c}24 \\
(80 \%)\end{array}$ & $\begin{array}{c}6 \\
(20 \%)\end{array}$ \\
\hline 2 & $\begin{array}{c}\text { X2. Statement } 2 \\
\text { Strive to regulate existing resources to } \\
\text { develop competitiveness and help } \\
\text { create the future }\end{array}$ & $\begin{array}{c}0 \\
(0)\end{array}$ & $\begin{array}{c}0 \\
(0)\end{array}$ & $\begin{array}{c}0 \\
(0)\end{array}$ & $\begin{array}{c}23 \\
(76,67 \%)\end{array}$ & $\begin{array}{c}7 \\
(23,3 \%)\end{array}$ \\
\hline 3 & $\begin{array}{c}\text { X2. Statement } 3 \\
\text { Implementation of Good Governance }\end{array}$ & $\begin{array}{c}0 \\
(0)\end{array}$ & $\begin{array}{c}0 \\
0 \\
(0)\end{array}$ & $\begin{array}{c}0 \\
0 \\
(0)\end{array}$ & $\begin{array}{c}25 \\
(83,3 \%)\end{array}$ & $\begin{array}{c}5 \\
(16,67 \%)\end{array}$ \\
\hline 4 & $\begin{array}{c}\text { X2. Statement } 4 \\
\text { Mengefektifkan fungsi internal audit. }\end{array}$ & $\begin{array}{c}0 \\
(0)\end{array}$ & $\begin{array}{c}0 \\
(0)\end{array}$ & $\begin{array}{c}0 \\
(0)\end{array}$ & $\begin{array}{c}26 \\
(86,67 \%)\end{array}$ & $\begin{array}{c}4 \\
(13,33 \%)\end{array}$ \\
\hline
\end{tabular}




\begin{tabular}{|c|c|c|c|c|c|c|}
\hline 5 & $\begin{array}{c}\text { X2. Statement } 5 \\
\text { Have a Vision and Mission Company. }\end{array}$ & $\begin{array}{c}0 \\
0 \\
(0)\end{array}$ & $\begin{array}{c}0 \\
(0)\end{array}$ & $\begin{array}{c}0 \\
(0)\end{array}$ & $\begin{array}{c}24 \\
(80 \%)\end{array}$ & $\begin{array}{c}6 \\
(20 \%)\end{array}$ \\
\hline 6 & $\begin{array}{c}\text { X2. Statement } 6 \\
\text { Conduct book closing activities / end- } \\
\text { month transactions in maximizing } \\
\text { existing transactions to be reported to } \\
\text { the head office }\end{array}$ & $\begin{array}{c}0 \\
(0)\end{array}$ & $\begin{array}{c}0 \\
(0)\end{array}$ & $\begin{array}{c}0 \\
(0)\end{array}$ & $\begin{array}{c}26 \\
(86,67 \%)\end{array}$ & $\begin{array}{c}4 \\
(13,33 \%)\end{array}$ \\
\hline \multirow{3}{*}{ No } & \multirow{3}{*}{ Information } & \multicolumn{5}{|c|}{ Score } \\
\hline & & $\begin{array}{l}\text { respondents } \\
\text { (SD) }\end{array}$ & $\begin{array}{l}\text { respondents } \\
\text { (D) }\end{array}$ & $\begin{array}{l}\text { respondents } \\
\text { (d) }\end{array}$ & $\begin{array}{l}\text { respondents } \\
\text { (A) }\end{array}$ & $\begin{array}{l}\text { respondents } \\
\text { (SA) }\end{array}$ \\
\hline & & 1 & 2 & 3 & 4 & 5 \\
\hline 7 & $\begin{array}{c}\text { X2. Statement } 7 \\
\text { Working together with insurance / other } \\
\text { companies to increase the company's } \\
\text { cash. }\end{array}$ & $\begin{array}{c}0 \\
(0)\end{array}$ & $\begin{array}{c}0 \\
(0)\end{array}$ & $\begin{array}{c}0 \\
(0)\end{array}$ & $\begin{array}{c}26 \\
(86,67 \%)\end{array}$ & $\begin{array}{c}4 \\
(13,33 \%)\end{array}$ \\
\hline 8 & $\begin{array}{l}\text { X2. Statement } 8 \\
\text { Billing the patient's receivables before } \\
\text { maturity so there will be no loss to the } \\
\text { company. }\end{array}$ & $\begin{array}{c}0 \\
(0)\end{array}$ & $\begin{array}{c}0 \\
(0)\end{array}$ & $\begin{array}{c}0 \\
(0)\end{array}$ & $\begin{array}{c}26 \\
(86,67 \%)\end{array}$ & $\begin{array}{c}4 \\
(13,33 \%)\end{array}$ \\
\hline 9 & $\begin{array}{c}\text { X2. Statement } 9 \\
\text { Have a good organizational structure }\end{array}$ & $\begin{array}{c}0 \\
(0)\end{array}$ & $\begin{array}{c}0 \\
(0)\end{array}$ & $\begin{array}{c}0 \\
(0)\end{array}$ & $\begin{array}{c}24 \\
(80 \%)\end{array}$ & $\begin{array}{c}6 \\
(20 \%)\end{array}$ \\
\hline 10 & $\begin{array}{c}\text { X2. Statement } 10 \\
\text { Ensure that the management of data } \\
\text { developed within the organization can } \\
\text { be trusted }\end{array}$ & $\begin{array}{c}0 \\
(0)\end{array}$ & $\begin{array}{c}0 \\
(0)\end{array}$ & $\begin{array}{c}3 \\
(10 \%)\end{array}$ & $\begin{array}{c}19 \\
(63,33 \%)\end{array}$ & $\begin{array}{c}8 \\
(36,67 \%)\end{array}$ \\
\hline 11 & $\begin{array}{c}\text { X2. Statement } 11 \\
\text {.Have a good accounting system }\end{array}$ & $\begin{array}{c}0 \\
(0)\end{array}$ & $\begin{array}{c}0 \\
(0)\end{array}$ & $\begin{array}{c}0 \\
(0)\end{array}$ & $\begin{array}{c}27 \\
(90 \%)\end{array}$ & $\begin{array}{c}3 \\
(10 \%)\end{array}$ \\
\hline 12 & $\begin{array}{c}\text { X2. Statement } 12 \\
\text { Compliance with company policies, } \\
\text { laws, regulations and standards. }\end{array}$ & $\begin{array}{c}0 \\
(0)\end{array}$ & $\begin{array}{c}0 \\
(0)\end{array}$ & $\begin{array}{c}3 \\
(10 \%)\end{array}$ & $\begin{array}{c}23 \\
(76,67 \%)\end{array}$ & $\begin{array}{c}4 \\
(13,33 \%)\end{array}$ \\
\hline \multirow[t]{2}{*}{13} & $\begin{array}{c}\text { X2. Statement } 13 \\
\text { Developing an attitude of commitment } \\
\text { to the company }\end{array}$ & $\begin{array}{c}0 \\
(0)\end{array}$ & $\begin{array}{c}0 \\
(0)\end{array}$ & $\begin{array}{c}0 \\
(0)\end{array}$ & $\begin{array}{c}28 \\
(93,33 \%)\end{array}$ & $\begin{array}{c}2 \\
(6,67 \%)\end{array}$ \\
\hline & $\begin{array}{l}\text { Average } \\
\end{array}$ & \multicolumn{4}{|c|}{$53,83 \%$} & Good \\
\hline
\end{tabular}

Source: processed data, 2018

\section{c. Finance Accounting Employee Performance (Y)}

Based on the data collected from the questionnaire about the performance of Employees finance accounting that the number of scores from these variables is shown in the following table.

Table 3.3. Score Y Respondents on the Questionnaire

\begin{tabular}{|c|c|c|c|c|c|c|}
\hline \multirow{3}{*}{ Number } & \multirow{3}{*}{ Information } & \multicolumn{5}{|c|}{ Score } \\
\hline & & $\begin{array}{l}\text { respondents } \\
\text { (SD) }\end{array}$ & $\begin{array}{l}\text { respondents } \\
\text { (D) }\end{array}$ & $\begin{array}{l}\text { respondents } \\
\text { (d) }\end{array}$ & $\begin{array}{l}\text { respondents } \\
\text { (A) }\end{array}$ & $\begin{array}{c}\text { respondents } \\
\text { (SA) }\end{array}$ \\
\hline & & 1 & 2 & 3 & 4 & 5 \\
\hline 1 & $\begin{array}{l}\text { Y. Statement } 1 \\
\text { Loyalitas dalam mengerjakan } \\
\text { pekerjaan. }\end{array}$ & $\begin{array}{c}0 \\
(0)\end{array}$ & $\begin{array}{c}0 \\
0\end{array}$ & $\begin{array}{c}1 \\
(3,33 \%)\end{array}$ & $\begin{array}{c}27 \\
(90 \%)\end{array}$ & $\begin{array}{c}2 \\
(6,67 \%)\end{array}$ \\
\hline 2 & $\begin{array}{c}\text { Y. Statement } 2 \\
\text { I work according to the } \\
\text { procedure. }\end{array}$ & $\begin{array}{c}0 \\
(0)\end{array}$ & $\begin{array}{c}0 \\
(0)\end{array}$ & $\begin{array}{c}1 \\
(3,33 \%)\end{array}$ & $\begin{array}{c}27 \\
(90 \%)\end{array}$ & $\begin{array}{c}2 \\
(6,67 \%)\end{array}$ \\
\hline \multirow[t]{2}{*}{3} & $\begin{array}{c}\text { Y. Statement } 3 \\
\text { I can account for the assignment }\end{array}$ & $\begin{array}{c}0 \\
(0)\end{array}$ & $\begin{array}{c}0 \\
(0)\end{array}$ & $\begin{array}{c}2 \\
(6,67 \%)\end{array}$ & $\begin{array}{c}25 \\
(86,66 \%)\end{array}$ & $\begin{array}{c}2 \\
(6,67 \%)\end{array}$ \\
\hline & & \multicolumn{5}{|c|}{ Score } \\
\hline
\end{tabular}




\begin{tabular}{|c|c|c|c|c|c|c|}
\hline Number & Information & $\begin{array}{l}\text { responden } \\
\text { (SD) }\end{array}$ & $\begin{array}{l}\text { responden } \\
\text { (D) }\end{array}$ & $\begin{array}{l}\text { responden } \\
\text { (d) }\end{array}$ & $\begin{array}{l}\text { responden } \\
\text { (A) }\end{array}$ & $\begin{array}{l}\text { responden } \\
\text { (SA) }\end{array}$ \\
\hline & & 1 & 2 & 3 & 4 & 5 \\
\hline 4 & $\begin{array}{c}\text { Y. Statement } 4 \\
\text { I was able to take the initiative in } \\
\text { work. }\end{array}$ & $\begin{array}{c}0 \\
(0)\end{array}$ & $\begin{array}{c}0 \\
(0)\end{array}$ & $\begin{array}{c}3 \\
(10 \%)\end{array}$ & $\begin{array}{c}26 \\
(86,67 \%)\end{array}$ & $\begin{array}{c}1 \\
(3,33 \%)\end{array}$ \\
\hline 5 & $\begin{array}{l}\text { Y. Statement } 5 \\
\text { Saya mampu menyelesaikan } \\
\text { setiap pekerjaan. }\end{array}$ & $\begin{array}{c}0 \\
(0)\end{array}$ & $\begin{array}{c}0 \\
0 \\
(0)\end{array}$ & $\begin{array}{c}3 \\
(10 \%)\end{array}$ & $\begin{array}{c}24 \\
(80 \%)\end{array}$ & $\begin{array}{c}3 \\
(10 \%)\end{array}$ \\
\hline 6 & $\begin{array}{c}\text { Y. Statement } 6 \\
\text { I always be polite to my boss }\end{array}$ & $\begin{array}{c}0 \\
(0)\end{array}$ & $\begin{array}{c}0 \\
(0)\end{array}$ & $\begin{array}{c}0 \\
(0)\end{array}$ & $\begin{array}{c}21 \\
(70 \%)\end{array}$ & $\begin{array}{c}9 \\
(30 \%)\end{array}$ \\
\hline 7 & $\begin{array}{c}\text { Y. Statement } 7 \\
\text { The work quantity of the } \\
\text { employees of this company is } \\
\text { better than the employees of other } \\
\text { companies }\end{array}$ & $\begin{array}{c}0 \\
(0)\end{array}$ & $\begin{array}{c}0 \\
(0)\end{array}$ & $\begin{array}{c}4 \\
(13,33 \%)\end{array}$ & $\begin{array}{c}22 \\
(73,34 \%)\end{array}$ & $\begin{array}{c}4 \\
(13,33 \%))\end{array}$ \\
\hline 8 & $\begin{array}{c}\text { Y. Statement } 8 \\
\text { The work quality of the } \\
\text { employees of this company is } \\
\text { better than the employees of other } \\
\text { companies. }\end{array}$ & $\begin{array}{c}0 \\
(0)\end{array}$ & $\begin{array}{c}0 \\
0 \\
(0)\end{array}$ & $\begin{array}{c}3 \\
(10 \%)\end{array}$ & $\begin{array}{c}22 \\
(73,34 \%)\end{array}$ & $\begin{array}{c}5 \\
(16,67 \%)\end{array}$ \\
\hline 9 & $\begin{array}{c}\text { Y. Statement } 9 \\
\text { Promoted by the leader to hold a } \\
\text { higher position, if he works } \\
\text { diligently. }\end{array}$ & $\begin{array}{c}0 \\
(0)\end{array}$ & $\begin{array}{c}0 \\
(0)\end{array}$ & $\begin{array}{c}6 \\
(20 \%)\end{array}$ & $\begin{array}{c}18 \\
(60 \%)\end{array}$ & $\begin{array}{c}6 \\
(20 \%)\end{array}$ \\
\hline 10 & $\begin{array}{c}\text { Y. Statement } 10 \\
\text { Do you agree to always improve } \\
\text { the quality of work which is } \\
\text { charged to you? }\end{array}$ & $\begin{array}{c}0 \\
(0)\end{array}$ & $\begin{array}{c}0 \\
(0)\end{array}$ & $\begin{array}{c}10 \\
(33,33 \%)\end{array}$ & $\begin{array}{c}18 \\
(60 \%)\end{array}$ & $\begin{array}{c}2 \\
(6,67 \%)\end{array}$ \\
\hline & Average & \multicolumn{4}{|c|}{$40,07 \%$} & Good \\
\hline
\end{tabular}

Source: processed data, 2018

\subsection{Quantitative Data Analysis}

\section{Preliminary Analysis}

Preliminary analysis in this study, namely the instrument test, to produce a measuring instrument, which is used in the form of a questionnaire has been able to measure each concept used. The quality test that the author uses, namely the validity test, reliability test, and normality test.

\section{Validity test}

Testing the validity of each item, is used with item analysis which correlates the score of each item with the total score, which is the number of each item score. In providing an interpretation of the correlation coefficient (r), items that have a positive correlation with the criterion (total score), as well as high correlation, indicate that the item has high validity as well. The statement item is declared valid if $r$ count $>r$ table. $(d f=n-2)>0.361$. For the number of respondents 30 used a correlation value of 0.361

Table 3.4. Validity Test

\begin{tabular}{|c|c|c|c|c|c|}
\hline $\begin{array}{c}\text { Num } \\
\text { ber }\end{array}$ & $\begin{array}{c}\text { Variable/ } \\
\text { Statement }\end{array}$ & Respondents & $\begin{array}{c}\text { Positive } \\
\text { Correlation }\end{array}$ & $\begin{array}{c}\text { Validity } \\
\text { Value } \\
\text { Standard }\end{array}$ & Information \\
\hline 1 & X1. Statement 1 & 30 & $0,617>$ & 0,361 & Valid \\
\hline 2 & X1. Statement 2 & 30 & $0,609>$ & 0,361 & Valid \\
\hline 3 & X1. Statement 3 & 30 & $0,417>$ & 0,361 & Valid \\
\hline
\end{tabular}




\begin{tabular}{|c|c|c|c|c|c|}
\hline 4 & X1. Statement 4 & 30 & $0,478>$ & 0,361 & Valid \\
\hline 5 & X1. Statement 5 & 30 & $0,636>$ & 0,361 & Valid \\
\hline 6 & X1. Statement 6 & 30 & $0,628>$ & 0,361 & Valid \\
\hline 7 & X1. Statement 7 & 30 & $0,610>$ & 0,361 & Valid \\
\hline 8 & X1. Statement 8 & 30 & $0,627>$ & 0,361 & Valid \\
\hline 9 & X1. Statement 9 & 30 & $0,806>$ & 0,361 & Valid \\
\hline 10 & X1. Statement 10 & 30 & $0,549>$ & 0,361 & Valid \\
\hline 11 & X1. Statement 11 & 30 & $0,540>$ & 0,361 & Valid \\
\hline 12 & X1. Statement 12 & 30 & $0,415>$ & 0,361 & Valid \\
\hline 13 & X1. Statement 13 & 30 & $0,703>$ & 0,361 & Valid \\
\hline 14 & X1. Statement 14 & 30 & $0,662>$ & 0,361 & Valid \\
\hline 15 & X1. Statement 15 & 30 & $0,601>$ & 0,361 & Valid \\
\hline 16 & X1. Statement 16 & 30 & $0,686>$ & 0,361 & Valid \\
\hline 17 & X1. Statement 17 & 30 & $0,488>$ & 0,361 & Valid \\
\hline 18 & X1. Statement 18 & 30 & $0,547>$ & 0,361 & Valid \\
\hline 19 & X2. Statement 1 & 30 & $0,578>$ & 0,361 & Valid \\
\hline 20 & X2. Statement 2 & 30 & $0,709>$ & 0,361 & Valid \\
\hline 21 & X2. Statement 3 & 30 & $0,643>$ & 0,361 & Valid \\
\hline 22 & X2. Statement 4 & 30 & $0,700>$ & 0,361 & Valid \\
\hline 23 & X2. Statement 5 & 30 & $0,728>$ & 0,361 & Valid \\
\hline 24 & X2. Statement 6 & 30 & $0,581>$ & 0,361 & Valid \\
\hline 25 & X2. Statement 7 & 30 & $0,522>$ & 0,361 & Valid \\
\hline 26 & X2. Statement 8 & 30 & $0,670>$ & 0,361 & Valid \\
\hline 27 & X2. Statement 9 & 30 & $0,653>$ & 0,361 & Valid \\
\hline $\begin{array}{c}\text { Num } \\
\text { ber }\end{array}$ & $\begin{array}{c}\text { Variable } \\
\text { Statement }\end{array}$ & Respondents & $\begin{array}{l}\text { Korelasi } \\
\text { Positive }\end{array}$ & $\begin{array}{l}\text { Standard } \\
\text { Validity } \\
\text { Value }\end{array}$ & Information \\
\hline 28 & X2. Statement 10 & 30 & $0,757>$ & 0,361 & Valid \\
\hline 29 & X2. Statement 11 & 30 & $0,553>$ & 0,361 & Valid \\
\hline 30 & X2. Statement 12 & 30 & $0,692>$ & 0,361 & Valid \\
\hline 31 & X2. Statement 13 & 30 & $0,577>$ & 0,361 & Valid \\
\hline 32 & Y. Statement 1 & 30 & $0,483>$ & 0,361 & Valid \\
\hline 33 & Y. Statement 2 & 30 & $0,632>$ & 0,361 & Valid \\
\hline 34 & Y. Statement 3 & 30 & $0,406>$ & 0,361 & Valid \\
\hline 35 & Y. Statement 4 & 30 & $0,560>$ & 0,361 & Valid \\
\hline 36 & Y. Statement 5 & 30 & $0,499>$ & 0,361 & Valid \\
\hline 37 & Y. Statement 6 & 30 & $0,728>$ & 0,361 & Valid \\
\hline 38 & Y. Statement 7 & 30 & $0,727>$ & 0,361 & Valid \\
\hline 39 & Y. Statement 8 & 30 & $0,776>$ & 0,361 & Valid \\
\hline 40 & Y. Statement 9 & 30 & $0,668>$ & 0,361 & Valid \\
\hline 41 & Y. Statement 10 & 30 & $0,646>$ & 0,361 & Valid \\
\hline
\end{tabular}

Source: processed data, 2018

\section{Reliability Test}

Reliability Test means to know whether the scale made in the questionnaire is consistent or not, for this reason, a consistent scale test is performed, using a scale reliability test, which will give an alpha coefficient that is between $0-1$, if the alpha coefficient, the value approaches one then it can be ascertained that the scale used in the questionnaire is internally consistent. The statement item is stated to be reliable if $r$ alpha $>0.600$. 
Table 3.5. Reliability Test

Reliability Statistics

\begin{tabular}{|c|c|c|c|c|}
\hline Variable & $\begin{array}{c}\text { Cronbach's } \\
\text { Alpha }\end{array}$ & $\begin{array}{c}\text { N of } \\
\text { Items }\end{array}$ & $\begin{array}{c}\text { Reliable } \\
\text { Alpha }\end{array}$ & Information \\
\hline $\mathrm{X}_{1}$ & $0,749>$ & 18 & 0,600 & Reliable \\
\hline $\mathrm{X}_{2}$ & $0,757>$ & 13 & 0,600 & Reliable \\
\hline $\mathrm{Y}$ & $0,751>$ & 10 & 0,600 & Reliable \\
\hline \multicolumn{2}{|c|}{ TOTAL } & 41 & \multicolumn{3}{c}{} \\
\hline
\end{tabular}

Source: processed data, 2018

Data reliability testing for the 41 questions below shows that the research instrument is reliable. With X1 $=0.749>0.600$ alpha; X2 $=$ $0.757>0.600$ alpha; and $\mathrm{Y}=0.751>0.600$ alpha.

\section{a. Multiple Linear Regression Test}

To find out the influence between organizational culture, and financial strategies on the performance of finance and accounting employees at PT Siloam International Hospitals Manado, multiple linear regression analysis is used, where the independent variable is Organizational Culture (X1), and financial strategy (X2), and the dependent variable is Finance and Acoounting employee performance $(\mathrm{Y})$. The following are the results of the tests carried out.

Table 3.6 Multiple Linear Regression Test Results

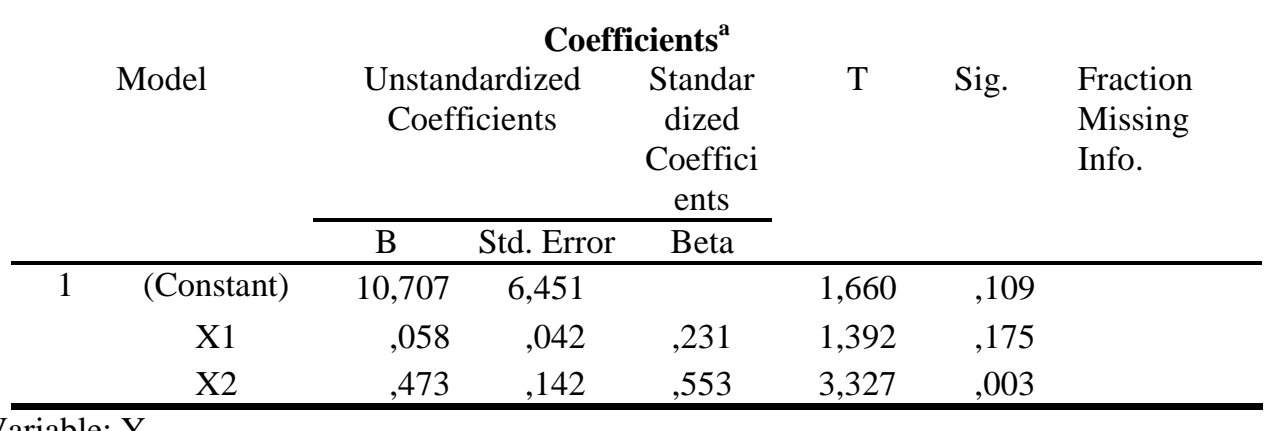

Dependent Variable: Y

Source: processed data, 2018

Multiple linear regression equation as follows:

$$
\mathbf{Y}=\mathbf{a}+\mathbf{b}_{1} \mathbf{X}_{1}+\mathbf{b}_{2} \mathbf{X}_{2}
$$

Where:

Y: Dependent Variables (Employee Performance)

a: Constant, that is the value of $\mathrm{Y}$ if $\mathrm{X} 1$ and $\mathrm{X} 2=0$

b1, b2: Regression coefficient, which is the value of increase or decrease

$\mathrm{Y}$ variable based on variables $\mathrm{X} 1$ and $\mathrm{X} 2$

$\mathrm{X} 1$ : Independent Variables (Organizational Culture)

X2: Independent Variables (Financial Strategy)

From the results of multiple regression tests performed, can be made an equation, as follows:

$$
\mathrm{Y}=10,707+0,058 \times 1+0,473 \times 2
$$

WWW.scirj.org

(C) 2018, Scientific Research Journal

http://dx.doi.org/10.31364/SCIRJ/v6.i9.2018.P0918560 
From the equation, the interpretation can be explained as follows:

a. a. Constant $(a)=10.707$, meaning that if the organizational culture variable (X1) and financial strategy (X2) are in a constant state, then the employee's performance $(\mathrm{Y})$ is 10.707 .

b. b. $\mathrm{b} 1=0.058$ means that if the financial strategy variable $(\mathrm{X} 1)$ increases by 1 unit, then employee performance (Y) will increase by 0.058 .

c. c. b2 $=0.473$ means that if the organizational culture variable (X2) increases by 1 unit, then employee performance (Y) will increase by 0.473 .

\section{b. Determination Coefficient}

The coefficient of determination (R2) is used to determine the contribution of independent variables in explaining the variation of the dependent variable. From table 3.7 it is obtained the coefficient of determination (R2) of 0.472 (47.2\%). This means that variations in the dependent variable finance employee performance, and accounting (Y) can be explained by organizational culture variables (X1), and financial strategies (X2) by $47.2 \%$, while the remaining $52.8 \%$ is explained by other variables outside the variables studied.

Table 3.7. Model Summary

\begin{tabular}{lllll}
\multicolumn{9}{c}{ Model Summary $^{\mathbf{b}}$} & \\
Model & $\mathrm{R}$ & R Square & Adjusted R Square & $\begin{array}{l}\text { Std. Error of the } \\
\text { Estimate }\end{array}$ \\
\hline 1 &, $713^{\mathrm{a}}$ &, 508 &, 472 & 2,09894 \\
\hline
\end{tabular}

Predictors: (Constant), X2, X1, Dependent Variable: Y

Source: processed data, 2018

c. F Test (Simultaneous Test)

Table 3.8.Uji F

ANOVA $^{\mathrm{a}}$

\begin{tabular}{ccccccc} 
& Model & $\begin{array}{c}\text { Sum of } \\
\text { Squares }\end{array}$ & Df & $\begin{array}{c}\text { Mean } \\
\text { Square }\end{array}$ & F & Sig. \\
\hline 1 & Regression & 122,917 & 2 & 61,458 & 13,950 &, $000^{\mathrm{b}}$ \\
& Residual & 118,950 & 27 & 4,406 & & \\
& Total & 241,867 & 29 & & & \\
\hline
\end{tabular}

a. Dependent Variable: Y

b. Predictors: (Constant), $\mathrm{X}_{2}, \mathrm{X}_{1}$

Source: processed data, 2018

This output explains the results of the F Test (regression coefficient test together), which is used to test the significance of the influence of several independent and dependent variables. In this case it is used to test the significance of the influence of organizational culture, and financial strategies together on the performance of finance and accounting employees, using a significant level of 5\% (0.05). For decision making, it can be seen the significance value (sig). If the significance is $<0.05$, then the conclusion is there is an influence between the variables of organizational culture and financial strategies on employee performance. If the significance is $>0.05$, there is no influence between organizational culture variables, and financial strategies on employee performance.

The significance value is $0,000<0,05$, so the conclusion is that there is a joint influence between organizational culture variables and financial strategies on the performance of finance employees and accounting.

\section{d. Partial T Test}

The $\mathrm{T}$ test is done by comparing the value of $\mathrm{t}$ count with $\mathrm{t}$ table or see the significance value. If $\mathrm{t}$ count $>\mathrm{t}$ table, and the significance value $<\alpha$ is 0.05 , it is said to have a significant effect, and if $\mathrm{t}$ count $<\mathrm{t}$ table, and the significance value $>\alpha 0.05$, then the effect is not significant. Obtained t count for organizational culture variable (X1) smaller $1.392<2.007$ and significance value $0.175>$ 
$\alpha 0.05$. So, organizational culture variables have no significant effect on the performance of employees of finance and accounting. While the $\mathrm{T}$ count for the financial strategy variable (X2) is greater than t table, namely 3.327> 2.007, and the significance value is $0.003<\alpha 0.05$. So, the financial strategy variable has an effect, and is significant on the performance of finance employees, and accounting (Y) partially.

The results of the $t$ test can be concluded that the most dominant variables influence the performance of finance employees and accounting at PT. Siloam International Hospitals Manado is a financial strategy variable with the largest $\mathrm{t}$ count value of 3.327 and the smallest significance value of 0.003 .

In testing the hypothesis the regression model uses the formula $\mathrm{n}-\mathrm{k}$ where:

$\mathrm{n}=$ Number of observations

$\mathrm{k}=$ Number of variables (free and bound)

So the number of respondents $30-3=27$

First hypothesis: Organizational Culture influences the performance of finance accounting employees. Test with a $=5 \%$

Second hypothesis: Organizational financial strategies affect the performance of finance accounting employees. Test with a $=5 \%$ $(0.05)$.

\section{e. Hypothesis Test Results}

Berdasarkan nilai t hitung dan t tabel dan nilai signifikan hasil output SPSS :

1. If $t$ counts $1.392<t$ table 1.703 , or the sig value is $0.175>0.05$ then $H 1$ : Denied (Does not affect $\mathrm{Y}$ ).

2. If $t$ counts $3.327>\mathrm{t}$ table 1.703 or sig value $0.003<0.05$, then $\mathrm{H} 2$ : Received (Affects $\mathrm{Y}$ ).

\section{f. Normality test}

Residual normality test with graphical method, that is by looking at the distribution of data on diagonal sources on the Normal P-P graph. Plots of standardized residual regression. As a basis for decision making if the points spread around the line and follow the diagonal line, the residual value is normal.

\section{Picture 3.1.}

\section{Normal P-P Plot of Regression Standardized Residual}

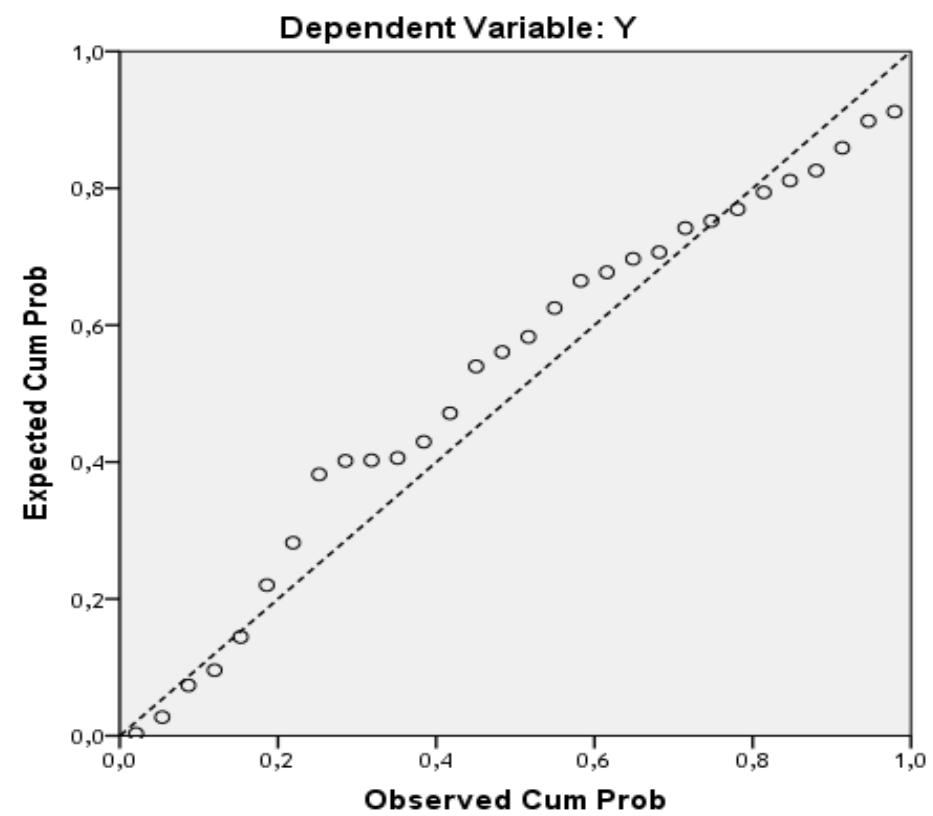

Source: processed data, 2018

Picture 3.1 indicates that the points spread around the line, and following the diagonal line the residual value is normal.

Picture 3.2. 


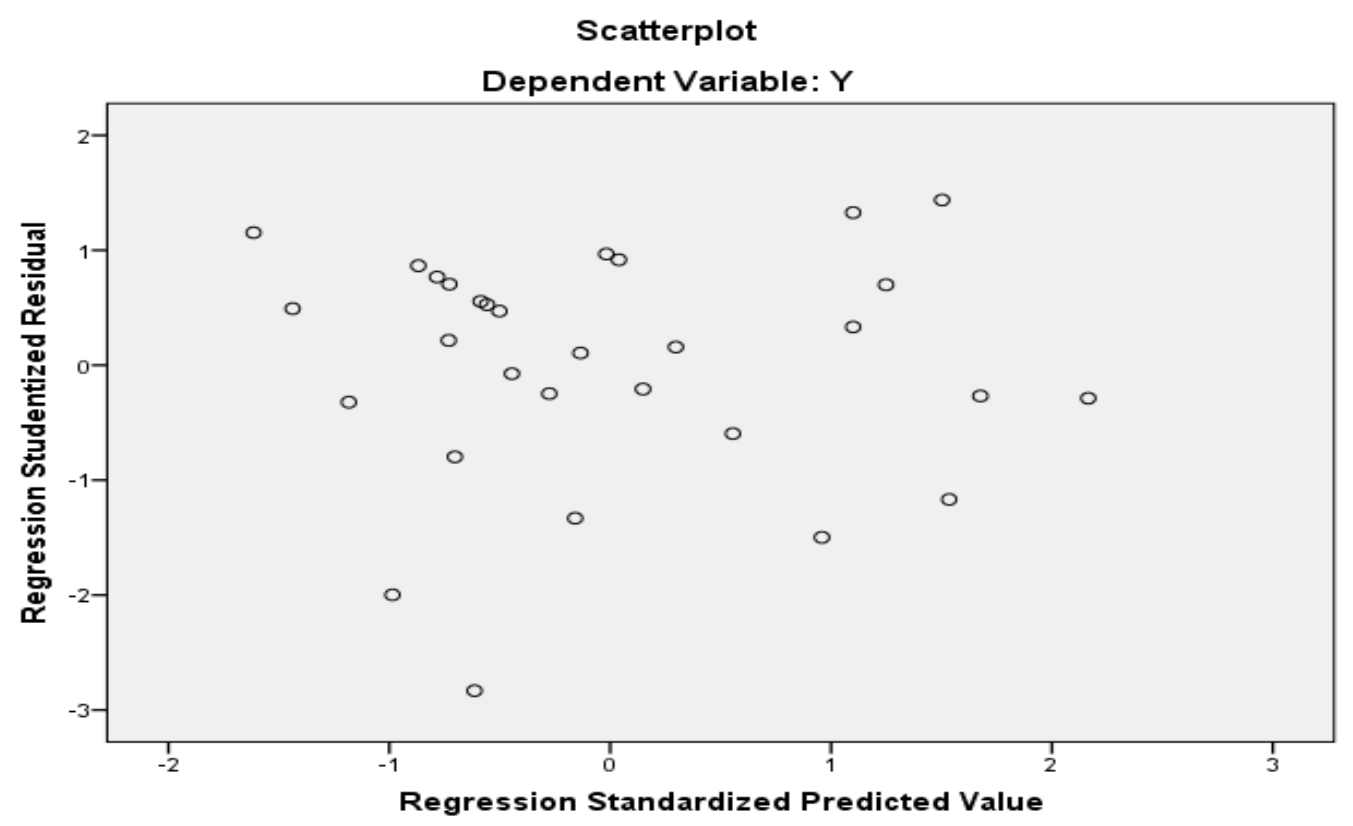

Source: processed data, 2018

Picture 3.2 used to detect heteroscedasticity. Heteroskedatisity is a residual variant that is not the same in all observations in the regression model. Good regression should not occur heteroscedasticity. The criteria, namely:

a. If there are certain patterns, such as dots that form a certain regular pattern (wavy, widened and then narrowed), heteroscedasticity occurs.

b. If there are no clear patterns, such as the spread points above, and below the 0 on the $\mathrm{Y}$ axis, heteroscedatisity does not occur. From the output can be known, that the points do not form a clear pattern (the points spread above, and below the number 0 on the $\mathrm{Y}$ axis). So it can be concluded that there is no heteroscedasticity in the regression model or it can be said that if the points spread then the variables of organizational culture and financial strategies are positively related.

Influence of Organizational Culture on Finance Accounting Employee Performance. Based on the results of the calculation of multiple linear regression analysis, with partial testing known, that the Organizational Culture variable (X1) has no positive effect (see table 3.6), a significant value greater than $0.175>0.05$. In table 3.6 obtained a coefficient of 0.058 , $t$-count 1.392 , and a significance value of 0.175 . That is, if the organizational culture variable is 1 unit, it will improve employee performance by 0.058 units. The performance of finance accounting employees will increase in line with the improvement of organizational culture at PT. Siloam International Hospitals Manado. Organizational culture can help employee performance, because it creates an extraordinary level of motivation, for employees to provide their best abilities, in utilizing the opportunities provided by their organizations. The shared values make employees feel comfortable working, committed, and loyal, and make employees try harder, improve employee performance and work, and maintain competitive advantage.

Financial Strategy Influence on the performance of finance accounting employees. The results of the calculation of multiple linear regression analysis, with partial testing it is known that the financial strategy variable (X2) has a positive effect (see table 3.6) smaller significant value $0.003<0.05$. In table 3.6 obtained a coefficient of 0.473 , $t$-count 3.327 , and a significance value of 0.003 which means if the financial strategy variable is 1 unit then it will improve employee performance by 0.473 units. In other words, the performance of finance accounting employees will increase in line with the correct financial strategy at PT. Siloam International Hospitals Manado.

\section{IV.CONCLUSION}

Based on the results of research at PT. Siloam International Hospitals Manado, it can be concluded that there is a positive influence, and significant financial strategy $0.003<0.05$, on employee performance in finance accounting, and there is a joint influence of $0.000<0.05$ between the variables of organizational culture, and financial strategy on the performance of finance accounting employees at PT Siloam Internasional Hospitals Manado partially. Financial Strategy is an action, or planning in increasing company profits. Financial planning is one part of the corporate planning organizational planning process. From the expected planning, the company can avoid mistakes, produce the best decisions, which ultimately can improve the performance of a company, so as not to happen accounting fraud. 


\section{REFERENCES}

Amrizal,2004,Pencegahan dan Pendektesian Kecurangan oleh Auditor Internal:Jakarta

Arep, Ishak dan Hendri, Tanjung. 2002, Manajemen Sumber Daya Manusia. Jakarta: Universitas Trisakti.

Black, Henry Campbell, Black's Law Dictionary, West Publishing Co., St. Paul, Minnessota, 1990, ed.6, hal. 339.

Budi, dan Waridin. 2006. Pengaruh Disiplin Kerja Karyawan dan Budaya Organisasi terhadap Kinerja di Divisi Radiologi RSUP

Dokter Kariadi Semarang, Vol. 2 No. 2, Hal. 181-250.

Dwi Priyatno;- Ed.I. 2012, Belajar Cepat Oleh Data Statistik dengan SPSS.:Yogyakarta

Eka Nuraini Rachmawati. 2004, Paradigma Baru Manajemen Sumber Daya Manusia Sebagai Basis Meraih Keunggulan Kompetitif. Yogyakarta: Ekonisia.

Gluck F. W., Kaufman S. P., and Wallack A. S., 1980, Strategic Management or Competitive Advantage, Harvard Business Review, Vol. 58, pp154-161.

Hax, A. C., and N. S. Majluf. 1996. The Strategy Concept and Process, A Pragmatic Approach, Prentice-Hall, Upper Saddle River, NJ.

G. Jack Bologna, Robert J. Lindquist dan Joseph T. Wells., Fraud Auditing and Forensic Accounting: New Tools and Techniques, (John Wiley \& Sons, Inc., 1995), hlm. 4.

Muhammad Zainur Roziqin. (2010). Kepuasan Kerja. Malang: Averroes Press

Robbins, Stephen P., 2006, Perilaku Keorganisasian Jilid 1 Edisi 9. Jakarta: PT Indeks kelompok Gramedia

Schein, E.H. 1992, Organizational Culture andLeadership. Penerbit: San Fransisco: JosseyBass.

Suyadi Prawirosentono (2008: 2, Management Sumber Daya Kebijakan Kinerja Karyawan. Yogjakarta : BPFE

Sugiyono, A.G. 2005. Metode penelitian kuantitatif, kualitatif, dan $R \&$ D. Alfabeta. Bandung.

Siti Munafiah. (2011). Pengaruh Kompensasi dan Supervisi terhadap Kinerja Karyawan (Studi Kasus pada PT. Industri Sandang Nusantara Unit Patal Secang). Skripsi. FE Universitas Negeri Yogyakarta.

Simanjuntak, Payaman J. 2005. Manajemen dan Evaluasi Kerja. Lembaga Penerbit FEUI, Jakarta.

Rival Veithzal. Manajemen Sumber Daya Manusia untuk Perusahaan : Dari Teori ke Praktik-Ed. 2-cet.4. Jakarta : Rajawali Pers, 2013.

Wheelen, T,L., dan Hunger, J.D. (1994) Strategic Managmenet and Business Policy, 4th Edition, Addison- Wesley Publishing Company, USA

Wilopo. 2006. “Analisis Faktor-faktor yang Berpengaruh Terhadap Kecenderungan Kecurangan Akuntansi : Studi Pada Perusahaan Publik dan Badan Usaha Milik Negara di Indonesia.” Simposium Nasional Akuntansi 9, K-AKPM 19. 